\title{
From cannabis to cannabidiol to treat epilepsy, where are we?
}

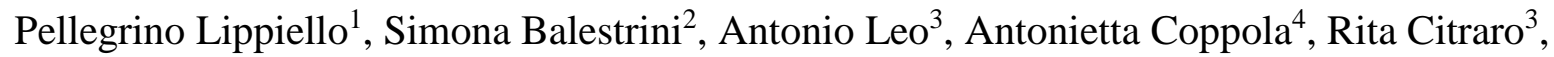
Maurizio Elia ${ }^{5}$,Emilio Russo ${ }^{3, *}$, Giovambattista De Sarro ${ }^{3}$

${ }^{1}$ Department of Pharmacy, University of Naples Federico II, Naples, Italy; ${ }^{2}$ NIHR University College London Hospitals Biomedical Research Centre, Department of Clinical and Experimental Epilepsy, UCL Institute of Neurology, London, UK, and Epilepsy Society, Chalfont St Peter, UK and Neuroscience Department, Polytechnic University of Marche, Ancona, Italy; ${ }^{3}$ Department Science of Health, School of Medicine and Surgery, University of Catanzaro, Italy; ${ }^{4}$ Epilepsy Centre, Department of Neuroscience, Odontostomatology and Reproductive Sciences, Federico II University, Naples, Italy; ${ }^{5}$ Oasi Institute for Research on Mental Retardation and Brain Aging (IRCCS), Troina (EN), Italy.

Running Title: Cannabidiol in epilepsy

\section{*Author for correspondence:}

Prof. Emilio Russo, PhD,

Chair of Pharmacology,

Department of Science of Health, School of Medicine,

University of Catanzaro, Italy

Via T. Campanella, 115; 88100 Catanzaro, ITALY.

Phone +39 0961 3694191; Fax +39 0961 3694192; e-mail: erusso@ unicz.it 


\section{Abstract}

Background: Several antiepileptic drugs (AEDs), about 25, are currently clinically available for the treatment of patients with epilepsy. Despite this armamentarium and the many recently introduced AEDs, no major advances have been achieved considering the number of drug resistant patients, while many benefits have been indeed obtained for other clinical outcomes (e.g. better tolerability, less interactions). Cannabinoids have long been studied for their potential therapeutical use and more recently phytocannabinoids have been considered a valuable tool for the treatment of several neurological disorders including epilepsy. Among this wide class, the most studied is cannabidiol (CBD) considering its lack of psychotropic effects and its anticonvulsant properties. Objective: Analyse the currently available literature on CBD also in light of other data on phytocannabinoids, reviewing data spanning from the mechanism of action, pharmacokinetic to clinical evidences. Results: Several preclinical studies have tried to understand the mechanism of action of CBD, which still remains largely not understood. CBD has shown significant anticonvulsant effects mainly in acute animal models of seizures; beneficial effects were reported also in animal models of epileptogenesis and chronic models of epilepsy, although not substantial. In contrast, data coming from some studies raise questions on the effects of other cannabinoids and above all marijuana. Conclusions: There is indeed sufficient supporting data for clinical development and important antiepileptic effects and the currently ongoing clinical studies will permit the real usefulness of CBD and possibly other cannabinoids. Undoubtedly, several issues also need to be addressed in the next future (e.g. better pharmacokinetic profiling). Finally, shading light on the mechanism of action and the study of other cannabinoids might represent an advantage for future developments.

Keywords: Cannabinoids; Cannabidiol; Epilepsy; Seizures; Preclinical studies; Clinical evidence. 


\section{Introduction}

Epilepsy is a severe neurological disorder of the central nervous system (CNS) characterized by spontaneous and recurrent seizures (SRS) [1-3]. Its etiology depends on the abnormal balance between excitatory and inhibitory cerebral activity leading to hyperexcitability $[4,5]$. However, neither basic mechanisms of seizures and even more epileptogenesis has been yet completely understood $[6,7]$. According to several hypotheses, epilepsy can be triggered by a brain insult, an infection, a genetic predisposition, but in some cases the onset of seizures is unprovoked and unpredictable [8,9]. Epilepsy patients are treated using antiepileptic drugs (AEDs) that are basically oriented to reduce the symptoms since for none of them disease modifying effects have been clinically proven $[6,10]$, furthermore, they strongly impact on patients quality of life according to their unavoidable burden of side effects $[11,12]$. The introduction of the new AEDs has also failed to improve the quality of life of epilepsy patients $[12,13]$. Therefore there is no drug able to prevent epilepsy or slow its progression. Nevertheless, it has been demonstrated that treatment during the early phases of the pathology might slow or modify the epileptogenic process and improve the quality of life of epilepsy patients [14]. For this reason, much effort of the researchers has been spent in the identification of new therapies also able to manage epileptogenesis [6] and new potential drugs effective in drug resistant patients [15-17]. Phytocannabinoids (terpenophenolic compounds synthesized by several cannabis species) have been recently reconsidered as a potential therapeutic strategy for epilepsy treatment based on old studies supporting their beneficial effects in neurological disorders including epilepsy [18-20]. Unfortunately, the clinical use of cannabinoid-based therapy still remains limited to only few pathologies [19]. In this article, we have summarized and reviewed the most relevant data on cannabidiol (CBD; representing the current leading compound of this group in epilepsy) spanning from 
the mechanism of action, pharmacokinetic to clinical evidences and keeping in mind the articles reporting phytocannabinoids effects in patients with epilepsy.

\section{CBD's pharmacokinetic profile in humans}

Several administration routes for CBD such as oral, transdermal, sublingual and inhalation have been examined and suggested. However, to date, several clinical studies in epilepsy, report that $\mathrm{CBD}$ has been mainly delivered orally through oil-based formulation, whereas the other administration routes, until now, have only demonstrated lacking importance in clinical practice. CBD after oral administration shows a variable pharmacokinetic profile, as a result of both low water solubility, which leads to an irregular gastrointestinal absorption, and of a significant first-pass metabolism [21]. Keeping with this, the CBD's oral bioavailability has been evaluated around 10\%. Moreover, following CBD oral administration, the time (Tmax) to reach the maximum plasma concentrations (Cmax) was 90-120 minutes [22]. At odds, the bioavailability of inhaled CBD, evaluated in subjects who smoked cannabis, has been around $31 \%$, whereas the Cmax has been reached in few minutes after smoking $(<10 \mathrm{~min})$ [23].

The distribution volume of CBD has been evaluated around $30 \mathrm{~L} / \mathrm{Kg}$. The high value of this volume is related with the high lipophilicity of $\mathrm{CBD}$, which is responsible for its fast distribution in fat organs including brain and adipose tissue [24]. Furthermore, CBD's affinity toward these organs leads to a half-life of about 24h (range: 18-32h) [25]. The plasma clearance determined after i.v. administration of CBD ranged from 960 to $1560 \mathrm{ml} / \mathrm{min}$. Moreover, CBD results to be highly bound to plasma proteins (> 99\%) and about $10 \%$ is bound to red blood cells [25].

CBD as well as several other cannabinoids is widely metabolized by cytochrome P450 (CYP450) enzymes in the liver. Particularly, the CYP3A and CYP2C are the isoform of enzymes mainly involved in CBD metabolisms. CBD's metabolites are mainly excreted in 
the feces, whereas only a low percentage results excreted in the urine [26]. Furthermore, it has been demonstrated that CBD is able to inhibit many classes of CYP450 enzymes including CYP3A and CYP2C families. This property of CBD leads to drug-drug interactions, which deserve to be further investigated [27]. In particular, drug-drug interaction between CBD and AEDs such as clobazam could also be used to manage refractory epilepsy considering that interactions do not necessarily have a negative impact if properly managed [28].

In this review, we have examined the literature about the effects of CBD treatment in epilepsy. For this purpose, we have considered both preclinical and clinical studies. A PubMed search for MEDLINE was performed to look for studies using the following keywords: "Cannabidiol and epilepsy", "Cannabidiol and seizure", "Cannabidiol and epileptogenesis", "Cannabidiol and animal model of epilepsy", "Cannabis and epilepsy", "Cannabis and seizure", "Cannabis and epileptogenesis" and "Cannabidiol and clinical studies". The date of our last search was April 20, 2016 and the period of time reviewed was approximately 40 years. Only English language papers were considered.

\section{Looking for CBD's mechanisms of action in epilepsy}

Cannabis contains many compounds, including at least 60 cannabinoids (about 100 is currently suggested) [29]. The two major neuroactive components are $\Delta 9$ tetrahydrocannabinol (THC) and the nonpsychotropic CBD. [21, 30]. Both CBD and THC have antiseizure effects. The antiseizure effect of THC is still controversial because of its pro- and anti-convulsant properties and the development of psychotropic and tolerance effects, due to these side effects, THC's clinical trials are limited. [31, 32]. CBD is used in both preclinical and clinical studies [33] since it has antiseizure activity with a good side- 
effect profile $[34,35]$ and no psychotropic effects. To date, the molecular mechanism of CBD action is not yet full understood [36].

The phytocannabinoids THC acts on the cannabinoid receptor type 1 (CB1) and cannabinoid receptor type 2 (CB2), while CBD has only a low affinity [36]. However, it has been demonstrated that $\mathrm{CBD}$ is able to antagonize $\mathrm{CB} 1$ and $\mathrm{CB} 2$ receptor agonists in vitro with unexpectedly high potency [37]. These properties of CBD might explain its lack of psychotropic effects $[38,39]$.

According to in vitro and in vivo assays, the therapeutic properties of CBD are independent by the endocannabinoid-signaling pathway and are likely mediated by enzymes, receptors, ion channels, and transporters $[21,36]$. However, should be noted that a dysregulation of the endocannabinoid-signaling pathway could play a key role in the pathogenesis of epilepsy including temporal lobe epilepsy [40, 41].

The possible molecular mechanisms by which CBD may influence neuronal hyperexcitability are: 1) activation of $5-\mathrm{HT}_{1 \mathrm{a}}$ receptors $[42,43]$; 2) reduction of the synaptic release of glutamate antagonizing $G$ protein-coupled receptor (GPR) 55); 3) stimulation and desensitization of transient receptor potential of vanilloid type 1 (TRPV1) and 2 (TRPV2) channels $[44,45]$; 4) inhibition of the synaptic uptake of GABA, noradrenaline, adenosine as well as dopamine [46, 47]; 5) stimulation of $\alpha 3$ and $\alpha 1$ glycine receptors [48, 49]; 6) stimulation and desensitization transient receptor potential of ankyrin type 1 (TRPA1) channel.

It has been hypothesized that $\mathrm{CBD}$ has also many other potential targets mediating its antiepileptic effects, such as: cyclooxygenase (COX), voltage-dependent anion channel 1 (VDAC1), nitric oxide (NO), G protein-coupled receptor (GPR) 18, peroxisome proliferatoractivated receptor $\gamma$ (PPAR- $\gamma$ ), tumor necrosis factor (TNF) and fatty acid amide hydrolase (FAAH). However, further studies are necessary to clarify the real efficacy of CBD on 
epilepsy patients and unravel its exact mechanism of action. Interestingly, CBD also possesses anxiolytic, antidepressant and antipsychotic properties that might be useful to treat psychiatric symptoms associated with epilepsy. The effects of CBD on cognitive functions represent another important therapeutical aspect to consider in the management of epilepsy patients [50-55].

Besides CBD, there are other cannabinoids that have shown an antiepileptic activity, both in in vitro assays and in vivo studies, such as cannabichromene (CBC), cannabidivarin (CBDV), and $\Delta 9$-tetrahydrocannabidivarin $(\triangle 9$-THCV); although there are no clinical studies proving their efficacy [21]. Among these compounds, the most interesting is currently CBDV, which is the propyl variant of cannabidiol $[22,56]$; CBDV has shown anticonvulsant properties through the involvement of specific molecular targets such as TRP channels or diacylglycerol lipase A [45, 57].

\section{Preclinical studies for CBD's antiepileptic effects}

In preclinical studies, CBD similarly to many other established AEDs has shown anticonvulsant activity in many acute animal models of seizures [21, 58]. It has been demonstrated that CBD pre-treatment was able to prevent tonic-seizures induced by GABA inhibitors, whereas it failed in case of seizures induced by the glycine antagonists strychnine [59]. On the other hand, CBD (60 mg/kg; i.p.) was not effective against focal seizures with secondary generalization induced by cobalt implantation [60].

Studies in the classically used animal models of epilepsy such as pentylenetetrazole (PTZ) and maximal electroshock (MES) have demonstrated that CBD better acts on tonic but not clonic seizures. In other words, this may suggest that CBD may act preferentially decreasing the spreading of seizures and partially influencing seizures onset [61, 62]. Moreover, CBD may increase the antiseizure activity of some AEDs (such as phenytoin) but might also 
decrease the effects of other AEDs (such as ethosuximide and clonazepam) as demonstrated in the MES model $[63,64]$. The drug-drug interaction among AEDs and CBD could be related to the ability of CBD to inhibit several isoforms of cytochrome P450 (CYP450), and this point still needs further clarification [27]. However, A clinical study Geffrey et al. [28]Geffrey et al. (2015)Geffrey et al. (2015)Geffrey et al. (2015)Geffrey et al. [28] has demonstrated that the association of CBD and clobazam (which are both catalyzed by CYP2C9 and CYP3A4 pathway) could be useful to treat children with refractory epilepsy (see Cannabis, cannabidiol and epilepsy: clinical data) [17].

By using the PTZ model of seizures, Jones et al. [65], have observed that a CBD pretreatment $(100 \mathrm{mg} / \mathrm{Kg}$; i.p. $)$ is able to decrease the occurrence of PTZ-induced seizures as well as rats mortality. $\mathrm{CBD}(0.01-100 \mu \mathrm{M})$ has shown antiepileptiform properties in hippocampal brain slices treated with $\mathrm{Mg}^{2+}$-free and 4-amynopyridine solutions, both displaying epileptic-like activity. The same study has also demonstrated that the mechanisms of actions of CBD in antiepileptiform activity in vitro and antiseizure activity in vivo are independent from CB1 receptor [65]. Further studies in a PTZ mouse model of seizure have shown that CBD pre-treatment (0.2-200 ng/mouse; i.c.v.) determines anticonvulsant effects, that are mitigated by co-administration of paxilline, an antagonist of voltage and $\mathrm{Ca}^{2+}$ activated $\mathrm{K}^{+}(\mathrm{BK})$ channels [49]. Likewise, in MES induced seizures, CBD pretreatment (20, 100, and $200 \mathrm{ng} / \mathrm{mouse}$; i.c.v.) induced anticonvulsant effects, which were not affected by co-administration of paxilline. These results suggest that the antiseizure effect of CBD, observed in PTZ model but not in other epilepsy models, could be linked to BK channels. [66, 67].

In a recent study, the antiseizure effects of CBD (10-50 mg/kg; i.v.) have been evaluated in a chronic model of epilepsy, which has been obtained by PTZ administration for 28 consecutive days. In this study, rats pre-treated with CBD (20 and 50mg/Kg) showed a 
reduction of PTZ-induced seizures, and a lower neuronal death in CA1 and CA3 hippocampal regions. The analysis of the morphological changes of hippocampal neurons, by immunohistochemistry and glial fibrillary acidic protein (GFAP), has shown that CBD is able to decrease hippocampal astrocytic hyperplasia after PTZ. Furthermore, the hippocampal expression of N-methyl-D-aspartic acid (NMDA) receptor subunit 1 is also reduced in rats treated with CBD [68].

CBD antiepileptic properties have also been tested in other animal models, such as temporal lobe epilepsy (the pilocarpine model) and partial epilepsy (penicillin model) [69]. In the pilocarpine rat model, CBD pre-treatment (1 and 100mg/kg; i.p.) decreased the occurrence of tonic-clonic seizures, without influencing the percentage of mortality. In the penicillin model of seizures, CBD treatment $(\geq 10 \mathrm{mg} / \mathrm{kg}$; i.p. $)$ reduced both tonic-clonic seizures and mortality. Another important aspect of this study is that CBD treatment has minimal side effects on motor performances at anticonvulsant doses as highlighted by several behavioral tests. Hosseinzadeh et al. [70] have also studied CBD's antiseizure/antiepileptogenic effects in the pilocarpine rat model. In this study the researchers have administered CBD in two different treatment schedules. The first group of rats received five consecutive days of CBD injections (100 ng; i.c.v.) started at the onset of the silent phase after pilocarpine-induced status epilepticus. The second group received a single injection of CBD (100 ng; i.c.v.) at the beginning of the chronic phase after the development of pilocarpine-SE induced chronic epilepsy. In both groups, CBD was able to reduce seizures. Moreover, in the silent phase, the repeated administrations of CBD delayed the onset of spontaneous recurrent seizures (SRS) probably by increasing autophagy and antioxidant defense in hippocampal cells, therefore suggesting possible antiepileptogenic effects [70]. This latter point is also supported by the demonstrated neuroprotective effect of CBD, which has been evaluated in hypoxic-ischemic (HI) newborn pigs. The administration of CBD $(0.1 \mathrm{mg} / \mathrm{kg}$, i.v $)$ prevented edema as well as 
seizures onset in newborn pigs after hypoxic-ischemic damage. Apparently, the neuroprotective effect of CBD, involved $\mathrm{CB} 2$ and 5HT1A receptors. In particular, it was suggested that $\mathrm{CBD}$ acts as an agonist of 5HT1A receptors, and as modulator of $\mathrm{CB} 2$ receptors by increasing the levels of endocannabinoids in the brain $[43,71,72]$.

CBDV, the propyl variant of CBD, has anticonvulsant activity as suggested by both in vitro assays and in vivo preclinical studies. Hill et al. [73] have reported antiseizure effects of CBDV (1-100 $\mu \mathrm{M})$ in hippocampal slice models of epilepsy induced by administration of 4$\mathrm{AP}$ and $\mathrm{Mg}^{2+}$-free solutions. In a further study, using the same $\mathrm{Mg}^{2+-}$ free model, it has been demonstrated that the antiepileptiform effects of CBDV could be related to an activation and desensitization of TRPV1 channels [45]. CBDV pre-treatment (1 hour before seizure induction) possesses not dose-dependent antiseizure properties in several animal models of epilepsy including PTZ (CBDV $=100 \mathrm{mg} / \mathrm{kg}$; i.p. $)$, audiogenic $(\mathrm{CBDV}=50 \mathrm{mg} / \mathrm{kg}$; i.p. $)$ and MES (CBDV $=100 \mathrm{mg} / \mathrm{kg}$; i.p.) [73]. At odds, in some studies, CBDV alone was not effective while it was able to potentiate AEDs effects. Briefly, CBDV (200 mg/kg; i.p.) has not shown any effects on pilocarpine-induced acute seizures, but it induces a decrease of seizures, only when combined with other AEDs such as phenobarbital or valproate [73]. CBDV (400 mg/kg, p.o.) effects on acute PTZ-induced seizures in rats was correlated to an increased expression of epilepsy-related genes such as Egr1, Fos, BDNF, Arc and Ccl4 [74]; CBD and CBDV might therefore differ both in their antiepileptic effects and their mechanisms of action.

Overall, CBD and CBDV possess antiepileptic efficacy in a variety of preclinical in vitro and in vivo models, which clearly supports their further development in clinical studies.

\section{Cannabis, cannabidiol and epilepsy: clinical data}

In contrast to the large available amount of preclinical data, the current supportive clinical evidence for the use of cannabinoids in human epilepsy is rather limited (Table 1). Most data 
concerning the clinical effects of marijuana in human patients have been gathered from anecdotal cases, phone or online surveys, epidemiological studies, and a few clinical trials.

The evidence extrapolated from anecdotal cases is not univocal. A small case series in 1949 reported treatment with a tetrahydrocannabinol (THC) homolog, up to $4 \mathrm{mg} / \mathrm{day}$, in five institutionalized children with refractory epilepsy and intellectual disability, with two of them achieving a significant improvement in seizure control [75]. A case report of a young man with epilepsy, having previously been seizure-free for six months, describes tonic-clonic seizure recurrence after frequent marijuana smoking over a three week-period [76]. Marihuana smoking, in conjunction with therapeutic doses of phenobarbital and diphenylhydantoin, was reported as effective in controlling seizures in one young case with epilepsy [77]. Another case report showed evidence of reduction of complex partial seizures following marijuana use $[77,78]$. A small case series on the therapeutic application of THC in children with various neurological conditions, including epilepsy and intellectual disability, showed some evidence of anticonvulsive effects. The doses ranged from 0.04 to $0.12 \mathrm{mg} / \mathrm{kg}$ body weight a day. In most cases treatment was discontinued and there was no any worsening following the withdrawal [79]. Two adult cases with focal epilepsy, with previously reported benefit from frequent marijuana smoking, experienced significant increase in seizure frequency, documented by video-EEG telemetry in the setting of marijuana discontinuation upon admission to the epilepsy monitoring unit [80]. A young girl with Dravet syndrome (SCN1A-positive) was started on adjunctive therapy with a high concentration of THC, given in conjunction with her prescribed clobazam, and had subsequent dramatic (>90\%) reduction of the frequency of her convulsive seizures. The effect had persisted for about 20 months, and she was been successfully weaned from her other antiepileptic drugs [81]. A 10-month-old boy, affected by malignant migrating partial seizures in infancy (MMPS), was started on adjunctive treatment with CBD (25 mg/mL at 10 
$\mathrm{mg} / \mathrm{kg} /$ day divided twice daily, up to $25 \mathrm{mg} / \mathrm{kg} /$ day twice daily). His seizure frequency decreased from 10-20 per day to five per week with up to nine days of clinical seizure freedom and he made some developmental gains [82]. Very recently, the use of CBD oil, in the management of an 18 year old male affected by super refractory status epilepticus (SRSE) in the setting of new-onset refractory status epilepticus (NORSE) syndrome, was reported. CBD oil was added to the current AEDs treatment regimen. CBD treatment (started at 2.5 $\mathrm{mg} / \mathrm{Kg} /$ day and increased up to a maximum of $24 \mathrm{mg} / \mathrm{Kg} /$ day) was not able to reduce focal and generalized seizures. Therefore, in agreement with both the family and clinical recommendation, AEDs and sedating drugs were reduced, whereas CBD oil administration continued. Unfortunately, the patient died after treatment withdrawal [83].

A telephone survey was conducted in 2001 in order to determine the prevalence of marijuana use and the beliefs regarding the effects of marijuana on seizures in patients with epilepsy. Of the 136 interviewed subjects, $28(21 \%)$ had used marijuana in the past year. In the logistic regression analysis, seizure frequency, longer disease duration, and other illicit drug use were predictors of frequent marijuana use. The majority of active users reported beneficial effects on seizures $(68 \%$ of active cannabis users reported reduced seizure severity, and $54 \%$ observed decreased seizure frequency). Twenty-four percent of all subjects believed marijuana was effective to treat epilepsy [84].

In USA, a survey was presented to parents belonging to a Facebook group dedicated to sharing information about the use of cannabidiol-enriched cannabis to treat their child's seizures. 16/19 parents reported a reduction in seizure frequency while taking medical marijuana; others reported beneficial effects like improved sleep and mood. Drowsiness and fatigue were reported by some parents. Thirteen children had Dravet syndrome, four had Doose syndrome, and one each had Lennox-Gastaut syndrome and idiopathic epilepsy [85]. A study, conducted between 2008 and 2010 at an epilepsy outpatient clinic in Berlin, aimed 
to assess the prevalence of cannabis and other illicit drug use among epilepsy patients and the effects of illicit drug use on epilepsy. They systematically interviewed 310 patients with epilepsy; of these, $63(20.3 \%)$ had consumed cannabis after their epilepsy was diagnosed. Most of them $(53,84.1 \%)$ did not observe any effect on the disease [86].

An online survey of parents who administered CBD-enriched cannabis preparations for the treatment of their children with epilepsy, including infantile spasms or Lennox-Gastaut syndrome, was conducted in 2014. Eighty-five percent of all parents reported a reduction in seizure frequency, and $14 \%$ reported complete seizure freedom. The median duration of CBD treatment was 6.8 months and the median dosage was $4.3 \mathrm{mg} / \mathrm{kg} / \mathrm{day}$. Also improvement in sleep, alertness, and mood during CBD treatment, was reported. The most frequent side effects were increased appetite and weight gain [87]. A retrospective chart review of children attending the neurology service at the Children's Hospital of Colorado included 75 patients or caregivers who had started taking oral cannabis extracts for refractory epilepsy. Of these, $57 \%$ reported improvement to either seizure duration or frequency. The responder rate varied based on the type of epilepsy: it was $23 \%$ in cases with Dravet syndrome, $0 \%$ in cases with Doose syndrome, and $89 \%$ in cases with Lennox-Gastaut syndrome. However, there was a discrepancy in responder rate between patients with preexisting residency in Colorado and those patients who had moved to Colorado seeking oral cannabis treatments, finding that the latter were more than 3 times as likely to report a $>50 \%$ seizure reduction than those who had a preexisting care in Colorado. Many of the families reported also improved behavior/alertness, language and motor skills. The most common adverse events were increased seizures or new seizures, somnolence/fatigue and GI symptoms [88].

A further online survey was conducted by the journal "Epilepsia" (official journal of the international league against epilepsy; ILAE) in 2014 to seek opinions about the use of medical marijuana and cannabidiol (CBD) for people with epilepsy. Of 776 who started the 
poll, $529(68 \%)$ completed all of the questions. A minority of epileptologists and general neurologists from Europe and North America said that there were sufficient safety (34\%) and efficacy (28\%) data to use medical marijuana for epilepsy. The survey asked: "Would you advise patients with severe, catastrophic epilepsy who have not responded to approved therapy to try medical marijuana?". The $48 \%$ of epileptologists and general neurologists said that such patients should be advised to try medical marijuana. Different opinions were gathered by patients and the public; almost all of them said that there were sufficient safety (96\%) and efficacy (95\%) data, and 98\% would recommend medical marijuana in cases of severe epilepsy. Also most general physicians, basic researchers, nurses, and allied health professions sided more with patients, replied that there were sufficient safety $(70 \%)$ and efficacy $(71 \%)$ data, and $83 \%$ said that severe cases should be advised to try medical marijuana. Regarding the question: "What is your opinion about having pharmacologic grade compounds containing CBD available to us in epilepsy patients?", the majority (78\%) responded: "Yes, such compounds should be available", with no differences comparing specialists, general medical personal, and patients and the public. This survey proves a wide diversity of opinion on the use of medical marijuana and CBD in epilepsy, with more general physicians, basic researchers, allied health personal, patients and the public, supporting its use compared with medical specialists [89].

A recent retrospective multicenter study described the effect of CBD-enriched medical cannabis on 74 children and adolescent with refractory epilepsy. The median duration of treatment was 5.5 months, with a follow-up of 10 months. The CBD dosage ranged from 1 to $20 \mathrm{mg} / \mathrm{kg} /$ die. Most of the patients (89\%) reported reduction in seizure frequency. Also among patients affected by epileptic encephalopathies with or without known genetic mutations, $66 \%$ had more than a $25 \%$ reduction in seizure frequency, with $51 \%$ reporting between 50 and $100 \%$ reduction in seizure frequency. In $7 \%$ of patients there was an 
aggravation of seizures, which led to CBD withdrawal. Improvement in behavior and alertness, language, communication, motor skills and sleep, is also reported. The most common adverse reactions were somnolence, fatigue, gastrointestinal disturbances and irritability [90].

Up to date, only a few placebo-controlled trials that involve the use of CBD as a treatment for epilepsy have been conducted. A Cochrane systematic review of randomized, controlled clinical trials was conducted over the use of CBD-enriched therapies for the treatment of human epilepsy [35]. They identified only four studies meeting their efficacy criteria, with a total sample of 48 participants in clinical studies, randomized to placebo or to $200-300 \mathrm{mg}$ of CBD per day [91-94].

In all four studies, patients with uncontrolled seizures were randomized into either placebo or CBD groups. The dose of CBD ranged from 200 to $300 \mathrm{mg}$. Of the four studies, two had reported a difference in seizure frequency between the two groups [91, 92]. In the first study, eight patients were included in the experimental group; of these, four became seizure-free during the treatment phase (8-18 weeks), three reported an improvement in seizure frequency, and one did not have any benefit. The clinical condition of the seven placebo patients remained unchanged whereas the condition of one patient clearly improved. Drowsiness was reported by four patients in the CBD-group [91]. In the second study, four patients were included in the add-on CBD-group vs five in the placebo group (on their habitual AED treatment). Two of the four patients became seizure-free for 12 months, one reported partial improvement in seizure frequency, and one did not show any clinical benefit. The clinical condition of the five placebo patients remained unchanged. No side effects were reported [92]. Two more recent studies suggested little or no difference in seizure frequency between placebo and CBD-groups $[93,94]$. One study included 12 patients with uncontrolled seizures and intellectual disability [93]. Few details are provided but no differences in seizure 
frequency between the two groups emerged. Mild drowsiness was reported as side effect. The other study was only reported in an International Conference on Cannabis and Cannabinoids: 12 patients were treated with placebo for six months followed by CBD or placebo for 12 months, with a cross-over design. No relevant reduction in seizure frequency was reported [94].

These trials do not provide sufficient and consistent data to prove the effectiveness of treatment with CBD in epilepsy. They only show short-term tolerability of CBD-enriched treatments, with only minor side effects such as drowsiness. However they do not provide data on the safety of long-term treatment with CBD as the exposure to CBD ranged from one to 12 months.

A systematic review to determine the efficacy of medical marijuana in several neurologic conditions concluded that, given the lack of Class I-III studies, data are insufficient to support or refute the efficacy of cannabinoids for treatment of epilepsy. Recently, clinical studies exploring the additive effects of CBD to usual AED regimens, in both children and adults, have significantly increased. In 2014, a prospective observational study described the efficacy of treatment with $25 \mathrm{mg} / \mathrm{kg} /$ day of CBD in addition to the habitual AEDs. Of 23 children and young adults with refractory epilepsy, nine had more than $50 \%$ reduction in seizure frequency $[95,96]$. Very recently, one clinical study showed a significant pharmacodynamics interaction between CBD and clobazam [28], requiring a clobazam dose reduction.

Fifteen clinical trials on the use of cannabinoids in epilepsy are currently registered in ClinialTrials.gov. Of these, eight studies use the plant-derived CBD, Epidiolex (GWP42003: GWPharmaceuticals), four studies use the synthetic CBD (Insys Therapeutics), and three studies use the propyl variant of CBD (GWP42006: GWPharmaceuticals). The studies that have been using Epidiolex include patients with refractory epilepsy, including Dravet, 
Lennox-Gastaut and Sturge-Weber syndromes. A recent multicentric open-label trial included patients with refractory childhood-onset epilepsy (including Dravet and LennoxGastaut syndromes), with stable AED doses before study entry. Patients were given oral cannabidiol at 2-5 mg/kg per day, up-titrated until a maximum dose of $25 \mathrm{mg} / \mathrm{kg}$ or $50 \mathrm{mg} / \mathrm{kg}$ per day. Of the 214 included patients, 137 (64\%) were included in the efficacy analysis and $162(76 \%)$ were included in the safety and tolerability analysis. For all seizure types, 51 $(37 \%)$ of patients had a reduction of $50 \%$ or more, $30(22 \%)$ patients had a response of $70 \%$ or more, and $11(8 \%)$ had a response of $90 \%$ or more. The median reduction in monthly motor seizures was 36.5\% (IQR 0-64.7). Adverse events were reported in 128 (79\%) of the patients within the safety group. The most common adverse events were: somnolence, decreased appetite, diarrhea, fatigue, and convulsion ( $\mathrm{n}=18$ [11\%]). Serious adverse events possibly related to cannabidiol use were reported in $20(12 \%)$ patients, the most common of which was status epilepticus. The results suggest moderate efficacy and adequate safety profile in patients with refractory epilepsy [95]. However this was an open label and uncontrolled study and both efficacy and safety need to be assessed through blinded, randomized controlled trials. As widely reported in literature, to provide reliable scientific evidence on efficacy and safety of $\mathrm{CBD}$, powered double-blind randomized, placebocontrolled clinical studies are needed [20]. Regarding to this unmet need, GW Pharmaceuticals has recently provided the preliminary results of CBD's effects (Epidiolex) against Dravet syndrome, in the first randomized phase 3 clinical trial. This study enrolled 120 patients (average age 10 years) affected by this severe myoclonic epilepsy of infancy. Of these patients, 61 were treated with $\mathrm{CBD}$ at $20 \mathrm{mg} / \mathrm{kg} / \mathrm{day}$, whereas 59 received placebo. CBD or placebo was co-administered together with the current AEDs treatment regimens. In this study, starting on the first month of treatment, CBD significantly reduced convulsive seizures in comparison to placebo. The anticonvulsive effect of CBD was maintained throughout the 
period of treatment. Furthermore, CBD resulted well tolerated in this clinical trial. The most common side effects were: somnolence, decreased appetite, diarrhea, fatigue, and convulsion (http://www.gwpharm.com).

\section{Conclusion}

Marijuana has long been used in traditional medicine or as a remedy for several pathologies despite its psychotropic effects; its effectiveness has been therefore recognized. Our current knowledge and technologies now permit to separate the various constituents of the plant extracts and identify their mechanisms of action and effects to be transferred to clinical practice following the standard drug development procedure. Considering the current need of more effective therapies in several clinical settings including epilepsy, this warrants further studies. Based on this background, several in vivo and in vitro studies have been recently performed to define CBD and other cannabinoids effects in epilepsy. CBD is indeed able to reduce seizures in a similar fashion than other AEDs in animal models; furthermore it seems to possess less side effects when compared to some other AEDs [58, 64]. Moreover, in different animal models, CBD has also demonstrated beneficial effects on cognitive performance as well as on mood disorders. These properties might also represent a further advantage in the treatment of patients with epilepsy considering that psychiatric comorbidities together with side effects affect quality of life and adherence more than seizures themselves [11, 12]. From the preclinical point of view, the main drawback concerning CBD is the scant information on its effects in chronic models of epilepsy while its effects on acute models of seizures are confirming its efficacy; furthermore, considering the neuroprotective effects that these drugs might possess, properly designed preclinical studies in epileptogenesis models are completely lacking.

The antiepileptic effects of CBD have been verified in patients with drug-resistant epilepsies; therefore the epileptologists, the neurologists and the families believed that CBD is a new and 
safer AED. CBD use can be also considered in some particular cases like "catastrophic" infancy and childhood epileptic syndromes, for example the above-mentioned Dravet, MMPS and Lennox-Gastaut syndromes. On the other hand, there are not sufficient studies in humans proving the efficacy for its use while its safety and tolerability is more convincingly determined [35]. Furthermore, the current knowledge on CBD pharmacokinetic and above all its effects on liver enzymes (i.e. induction or inhibition) is not completely clear.

Data on patients with epilepsy are scarce and somehow difficult to interpret considering that different cannabinoid preparations were used in the trials, the optimal doses and the safety profile were also not always clearly defined. Another important aspect is that there are not sufficient studies regarding the effects (also adverse) related to the long-term use of the drug, such as motor dysfunctions, psychiatric disorders, impairment of neuropsychological functions and risk of addiction [18]. In order to address these issues, placebo-controlled, double-blind and multicentered clinical trials, using a relevant number of patients with similar clinical backgrounds, are necessary to have a better knowledge of the efficacy and safety use of CBD and derivatives. Finally, it will be extremely relevant to further study other cannabinoids and their mechanisms of action in order to use this data as a springboard for the development of effective drugs in epilepsy and other human diseases.

\section{Conflict of interest}

There are no conflicts of interest to be disclosed

\section{Acknowledgements}

None 


\section{References}

[1] Ben-Menachem E. Medical management of refractory epilepsy--practical treatment with novel antiepileptic drugs. Epilepsia. 2014;55 Suppl 1:3-8.

[2] Glauser T, Ben-Menachem E, Bourgeois B, et al. Updated ILAE evidence review of antiepileptic drug efficacy and effectiveness as initial monotherapy for epileptic seizures and syndromes. Epilepsia. 2013;54:551-63.

[3] Korff CM, Scheffer IE. Epilepsy classification: a cycle of evolution and revolution. Curr Opin Neurol. 2013;26:163-7.

[4] Scharfman HE. The neurobiology of epilepsy. Current neurology and neuroscience reports. 2007;7:348-54.

[5] Galanopoulou AS. Basic mechanisms of catastrophic epilepsy -- overview from animal models. Brain \& development. 2013;35:748-56.

[6] Pitkanen A, Engel J, Jr. Past and present definitions of epileptogenesis and its biomarkers. Neurotherapeutics. 2014;11:231-41.

[7] Jehi LE, Vezzani A. Novel concepts in epileptogenesis and its prevention. Neurotherapeutics. 2014;11:229-30.

[8] Goldberg EM, Coulter DA. Mechanisms of epileptogenesis: a convergence on neural circuit dysfunction. Nat Rev Neurosci. 2013;14:337-49.

[9] Pitkanen A, Lukasiuk K. Mechanisms of epileptogenesis and potential treatment targets. Lancet neurology. 2011;10:173-86.

[10] Trinka E, Brigo F. Antiepileptogenesis in humans: disappointing clinical evidence and ways to move forward. Curr Opin Neurol. 2014;27:227-35. 
[11] Luoni C, Bisulli F, Canevini MP, et al. Determinants of health-related quality of life in pharmacoresistant epilepsy: results from a large multicenter study of consecutively enrolled patients using validated quantitative assessments. Epilepsia. 2011;52:2181-91.

[12] Perucca P, Gilliam FG. Adverse effects of antiepileptic drugs. Lancet neurology. $2012 ; 11: 792-802$

[13] Kwan P, Brodie MJ. Refractory epilepsy: mechanisms and solutions. Expert review of neurotherapeutics. 2006;6:397-406.

[14] White HS, Loscher W. Searching for the ideal antiepileptogenic agent in experimental models: single treatment versus combinatorial treatment strategies. Neurotherapeutics. 2014;11:373-84.

[15] Laxer KD, Trinka E, Hirsch LJ, et al. The consequences of refractory epilepsy and its treatment. Epilepsy Behav. 2014;37:59-70.

[16] Franco V, Canevini MP, Capovilla G, et al. Off-label prescribing of antiepileptic drugs in pharmacoresistant epilepsy: a cross-sectional drug utilization study of tertiary care centers in Italy. CNS Drugs. 2014;28:939-49.

[17] Santulli L, Coppola A, Balestrini S, Striano S. The challenges of treating epilepsy with 25 antiepileptic drugs. Pharmacological research. 2016;107:211-9.

[18] Friedman D, Devinsky O. Cannabinoids in the Treatment of Epilepsy. N Engl J Med. $2015 ; 373: 1048-58$.

[19] Rosenberg EC, Tsien RW, Whalley BJ, Devinsky O. Cannabinoids and Epilepsy. Neurotherapeutics. 2015;12:747-68. 
[20] Leo A, Russo E, Elia M. Cannabidiol and epilepsy: Rationale and therapeutic potential. Pharmacological research. 2016;107:85-92.

[21] Devinsky O, Cilio MR, Cross $\mathrm{H}$, et al. Cannabidiol: pharmacology and potential therapeutic role in epilepsy and other neuropsychiatric disorders. Epilepsia. 2014;55:791-802.

[22] Bialer M, Johannessen SI, Levy RH, Perucca E, Tomson T, White HS. Progress report on new antiepileptic drugs: A summary of the Twelfth Eilat Conference (EILAT XII). Epilepsy Res. 2015;111:85-141.

[23] Ohlsson A, Lindgren JE, Andersson S, Agurell S, Gillespie H, Hollister LE. Single-dose kinetics of deuterium-labelled cannabidiol in man after smoking and intravenous administration. Biomedical \& environmental mass spectrometry. 1986;13:77-83.

[24] Castaneto MS, Wohlfarth A, Desrosiers NA, Hartman RL, Gorelick DA, Huestis MA. Synthetic cannabinoids pharmacokinetics and detection methods in biological matrices. Drug metabolism reviews. 2015;47:124-74.

[25] Huestis MA. Pharmacokinetics and metabolism of the plant cannabinoids, delta9tetrahydrocannabinol, cannabidiol and cannabinol. Handbook of experimental pharmacology. 2005:657-90.

[26] McGilveray IJ. Pharmacokinetics of cannabinoids. Pain research \& management : the journal of the Canadian Pain Society = journal de la societe canadienne pour le traitement de la douleur. 2005;10 Suppl A:15A-22A.

[27] Zendulka O, Dovrtelova G, Noskova K, et al. Cannabinoids and Cytochrome P450 Interactions. Current drug metabolism. 2016;17:206-26. 
[28] Geffrey AL, Pollack SF, Bruno PL, Thiele EA. Drug-drug interaction between clobazam and cannabidiol in children with refractory epilepsy. Epilepsia. 2015;56:1246-51.

[29] Galal AM, Slade D, Gul W, El-Alfy AT, Ferreira D, Elsohly MA. Naturally occurring and related synthetic cannabinoids and their potential therapeutic applications. Recent patents on CNS drug discovery. 2009;4:112-36.

[30] Katona I. Cannabis and Endocannabinoid Signaling in Epilepsy. Handbook of experimental pharmacology. 2015;231:285-316.

[31] Killestein J. Cannabinoids in the Treatment of Epilepsy. N Engl J Med. 2016;374:94.

[32] Szaflarski JP, Bebin EM. Cannabis, cannabidiol, and epilepsy--from receptors to clinical response. Epilepsy Behav. 2014;41:277-82.

[33] Paolino MC, Ferretti A, Papetti L, Villa MP, Parisi P. Cannabidiol as potential treatment in refractory pediatric epilepsy. Expert review of neurotherapeutics. 2016;16:17-21.

[34] Detyniecki K, Hirsch LJ. Cannabidiol for epilepsy: trying to see through the haze. Lancet neurology. 2016;15:235-7.

[35] Gloss D, Vickrey B. Cannabinoids for epilepsy. The Cochrane database of systematic reviews. 2014;3:CD009270.

[36] Ibeas Bih C, Chen T, Nunn AV, Bazelot M, Dallas M, Whalley BJ. Molecular Targets of Cannabidiol in Neurological Disorders. Neurotherapeutics. 2015;12:699-730.

[37] Thomas A, Baillie GL, Phillips AM, Razdan RK, Ross RA, Pertwee RG. Cannabidiol displays unexpectedly high potency as an antagonist of CB1 and CB2 receptor agonists in vitro. British journal of pharmacology. 2007;150:613-23. 
[38] McPartland JM, Duncan M, Di Marzo V, Pertwee RG. Are cannabidiol and Delta(9) tetrahydrocannabivarin negative modulators of the endocannabinoid system? A systematic review. British journal of pharmacology. 2015;172:737-53.

[39] Pertwee RG. The diverse $\mathrm{CB} 1$ and $\mathrm{CB} 2$ receptor pharmacology of three plant cannabinoids: delta9-tetrahydrocannabinol, cannabidiol and delta9-tetrahydrocannabivarin. British journal of pharmacology. 2008;153:199-215.

[40] Romigi A, Bari M, Placidi F, et al. Cerebrospinal fluid levels of the endocannabinoid anandamide are reduced in patients with untreated newly diagnosed temporal lobe epilepsy. Epilepsia. 2010;51:768-72.

[41] Goffin K, Van Paesschen W, Van Laere K. In vivo activation of endocannabinoid system in temporal lobe epilepsy with hippocampal sclerosis. Brain : a journal of neurology. $2011 ; 134: 1033-40$.

[42] Russo EB, Burnett A, Hall B, Parker KK. Agonistic properties of cannabidiol at 5-HT1a receptors. Neurochemical research. 2005;30:1037-43.

[43] Pazos MR, Mohammed N, Lafuente $\mathrm{H}$, et al. Mechanisms of cannabidiol neuroprotection in hypoxic-ischemic newborn pigs: role of 5HT(1A) and CB2 receptors. Neuropharmacology. 2013;71:282-91.

[44] De Petrocellis L, Ligresti A, Moriello AS, et al. Effects of cannabinoids and cannabinoid-enriched Cannabis extracts on TRP channels and endocannabinoid metabolic enzymes. British journal of pharmacology. 2011;163:1479-94.

[45] Iannotti FA, Hill CL, Leo A, et al. Nonpsychotropic Plant Cannabinoids, Cannabidivarin (CBDV) and Cannabidiol (CBD), Activate and Desensitize Transient Receptor Potential 
Vanilloid 1 (TRPV1) Channels in Vitro: Potential for the Treatment of Neuronal Hyperexcitability. ACS chemical neuroscience. 2014.

[46] Pandolfo P, Silveirinha V, dos Santos-Rodrigues A, et al. Cannabinoids inhibit the synaptic uptake of adenosine and dopamine in the rat and mouse striatum. European journal of pharmacology. 2011;655:38-45.

[47] Banerjee SP, Snyder SH, Mechoulam R. Cannabinoids: influence on neurotransmitter uptake in rat brain synaptosomes. The Journal of pharmacology and experimental therapeutics. 1975;194:74-81.

[48] Xiong W, Cui T, Cheng K, et al. Cannabinoids suppress inflammatory and neuropathic pain by targeting alpha3 glycine receptors. The Journal of experimental medicine. 2012;209:1121-34.

[49] Ahrens J, Demir R, Leuwer M, et al. The nonpsychotropic cannabinoid cannabidiol modulates and directly activates alpha-1 and alpha-1-Beta glycine receptor function. Pharmacology. 2009;83:217-22.

[50] Blessing EM, Steenkamp MM, Manzanares J, Marmar CR. Cannabidiol as a Potential Treatment for Anxiety Disorders. Neurotherapeutics. 2015;12:825-36.

[51] Micale V, Di Marzo V, Sulcova A, Wotjak CT, Drago F. Endocannabinoid system and mood disorders: priming a target for new therapies. Pharmacology \& therapeutics. 2013;138:18-37.

[52] Manseau MW, Goff DC. Cannabinoids and Schizophrenia: Risks and Therapeutic Potential. Neurotherapeutics. 2015;12:816-24. 
[53] Brooks-Kayal AR, Bath KG, Berg AT, et al. Issues related to symptomatic and diseasemodifying treatments affecting cognitive and neuropsychiatric comorbidities of epilepsy. Epilepsia. 2013;54 Suppl 4:44-60.

[54] Kanner AM, Barry JJ, Gilliam F, Hermann B, Meador KJ. Depressive and anxiety disorders in epilepsy: do they differ in their potential to worsen common antiepileptic drugrelated adverse events? Epilepsia. 2012;53:1104-8.

[55] Linge R, Jimenez-Sanchez L, Campa L, et al. Cannabidiol induces rapid-acting antidepressant-like effects and enhances cortical 5-HT/glutamate neurotransmission: role of 5-HT1A receptors. Neuropharmacology. 2016;103:16-26.

[56] Hill AJ, Williams CM, Whalley BJ, Stephens GJ. Phytocannabinoids as novel therapeutic agents in CNS disorders. Pharmacology \& therapeutics. 2012;133:79-97.

[57] Hill TD, Cascio MG, Romano B, et al. Cannabidivarin-rich cannabis extracts are anticonvulsant in mouse and rat via a CB1 receptor-independent mechanism. British journal of pharmacology. 2013;170:679-92.

[58] dos Santos RG, Hallak JE, Leite JP, Zuardi AW, Crippa JA. Phytocannabinoids and epilepsy. Journal of clinical pharmacy and therapeutics. 2015;40:135-43.

[59] Consroe P, Benedito MA, Leite JR, Carlini EA, Mechoulam R. Effects of cannabidiol on behavioral seizures caused by convulsant drugs or current in mice. European journal of pharmacology. 1982;83:293-8.

[60] Colasanti BK, Lindamood C, 3rd, Craig CR. Effects of marihuana cannabinoids on seizure activity in cobalt-epileptic rats. Pharmacology, biochemistry, and behavior. $1982 ; 16: 573-8$. 
[61] Karler R, Turkanis SA. The cannabinoids as potential antiepileptics. Journal of clinical pharmacology. 1981;21:437S-48S.

[62] Carlini EA, Leite JR, Tannhauser M, Berardi AC. Letter: Cannabidiol and Cannabis sativa extract protect mice and rats against convulsive agents. The Journal of pharmacy and pharmacology. 1973;25:664-5.

[63] Consroe P, Wolkin A. Cannabidiol--antiepileptic drug comparisons and interactions in experimentally induced seizures in rats. The Journal of pharmacology and experimental therapeutics. 1977;201:26-32.

[64] Scuderi C, Filippis DD, Iuvone T, Blasio A, Steardo A, Esposito G. Cannabidiol in medicine: a review of its therapeutic potential in CNS disorders. Phytotherapy research : PTR. 2009;23:597-602.

[65] Jones NA, Hill AJ, Smith I, et al. Cannabidiol displays antiepileptiform and antiseizure properties in vitro and in vivo. The Journal of pharmacology and experimental therapeutics. 2010;332:569-77.

[66] Leo A, Citraro R, Constanti A, De Sarro G, Russo E. Are big potassium-type Ca(2+)activated potassium channels a viable target for the treatment of epilepsy? Expert opinion on therapeutic targets. 2015;19:911-26.

[67] Shirazi-zand Z, Ahmad-Molaei L, Motamedi F, Naderi N. The role of potassium BK channels in anticonvulsant effect of cannabidiol in pentylenetetrazole and maximal electroshock models of seizure in mice. Epilepsy Behav. 2013;28:1-7. 
[68] Mao K, You C, Lei D, Zhang H. High dosage of cannabidiol (CBD) alleviates pentylenetetrazole-induced epilepsy in rats by exerting an anticonvulsive effect. International journal of clinical and experimental medicine. 2015;8:8820-7.

[69] Jones NA, Glyn SE, Akiyama S, et al. Cannabidiol exerts anti-convulsant effects in animal models of temporal lobe and partial seizures. Seizure. 2012;21:344-52.

[70] Hosseinzadeh M, Nikseresht S, Khodagholi F, Naderi N, Maghsoudi N. Cannabidiol Post-Treatment Alleviates Rat Epileptic-Related Behaviors and Activates Hippocampal Cell Autophagy Pathway Along with Antioxidant Defense in Chronic Phase of PilocarpineInduced Seizure. Journal of molecular neuroscience : MN. 2016;58:432-40.

[71] Alvarez FJ, Lafuente H, Rey-Santano MC, et al. Neuroprotective effects of the nonpsychoactive cannabinoid cannabidiol in hypoxic-ischemic newborn piglets. Pediatric research. 2008;64:653-8.

[72] Hillard CJ. Role of cannabinoids and endocannabinoids in cerebral ischemia. Current pharmaceutical design. 2008;14:2347-61.

[73] Hill AJ, Mercier MS, Hill TD, et al. Cannabidivarin is anticonvulsant in mouse and rat. British journal of pharmacology. 2012;167:1629-42.

[74] Amada N, Yamasaki Y, Williams CM, Whalley BJ. Cannabidivarin (CBDV) suppresses pentylenetetrazole (PTZ)-induced increases in epilepsy-related gene expression. PeerJ. 2013;1:e214.

[75] J.P. Davis HHR. Anti-epileptic action of marijuana- active substances. Federation Proceedings. 1949;8:167. 
[76] Keeler MH, Reifler CB. Grand mal convulsions subsequent to marijuana use. Case report. Diseases of the nervous system. 1967;28:474-5.

[77] Consroe PF, Wood GC, Buchsbaum H. Anticonvulsant nature of marihuana smoking. JAMA : the journal of the American Medical Association. 1975;234:306-7.

[78] Ellison JM, Gelwan E, Ogletree J. Complex partial seizure symptoms affected by marijuana abuse. The Journal of clinical psychiatry. 1990;51:439-40.

[79] Lorenz R. On the application of cannabis in paediatrics and epileptology. Neuro endocrinology letters. 2004;25:40-4.

[80] Hegde M, Santos-Sanchez C, Hess CP, Kabir AA, Garcia PA. Seizure exacerbation in two patients with focal epilepsy following marijuana cessation. Epilepsy Behav. 2012;25:563-6.

[81] Maa E, Figi P. The case for medical marijuana in epilepsy. Epilepsia. 2014;55:783-6.

[82] Saade D, Joshi C. Pure cannabidiol in the treatment of malignant migrating partial seizures in infancy: a case report. Pediatric neurology. 2015;52:544-7.

[83] Rosemergy I, Adler J, Psirides A. Cannabidiol oil in the treatment of super refractory status epilepticus. A case report. Seizure. 2016;35:56-8.

[84] Gross DW, Hamm J, Ashworth NL, Quigley D. Marijuana use and epilepsy: prevalence in patients of a tertiary care epilepsy center. Neurology. 2004;62:2095-7.

[85] Porter BE, Jacobson C. Report of a parent survey of cannabidiol-enriched cannabis use in pediatric treatment-resistant epilepsy. Epilepsy Behav. 2013;29:574-7. 
[86] Hamerle M, Ghaeni L, Kowski A, Weissinger F, Holtkamp M. Cannabis and other illicit drug use in epilepsy patients. European journal of neurology. 2014;21:167-70.

[87] Hussain SA, Zhou R, Jacobson C, et al. Perceived efficacy of cannabidiol-enriched cannabis extracts for treatment of pediatric epilepsy: A potential role for infantile spasms and Lennox-Gastaut syndrome. Epilepsy Behav. 2015;47:138-41.

[88] Press CA, Knupp KG, Chapman KE. Parental reporting of response to oral cannabis extracts for treatment of refractory epilepsy. Epilepsy Behav. 2015;45:49-52.

[89] Mathern GW, Beninsig L, Nehlig A. Fewer specialists support using medical marijuana and CBD in treating epilepsy patients compared with other medical professionals and patients: result of Epilepsia's survey. Epilepsia. 2015;56:1-6.

[90] Tzadok M, Uliel-Siboni S, Linder I, et al. CBD-enriched medical cannabis for intractable pediatric epilepsy: The current Israeli experience. Seizure. 2016;35:41-4.

[91] Cunha JM, Carlini EA, Pereira AE, et al. Chronic administration of cannabidiol to healthy volunteers and epileptic patients. Pharmacology. 1980;21:175-85.

[92] Mechoulam R, Carlini EA. Toward drugs derived from cannabis. Die Naturwissenschaften. 1978;65:174-9.

[93] Ames FR, Cridland S. Anticonvulsant effect of cannabidiol. South African medical journal $=$ Suid-Afrikaanse tydskrif vir geneeskunde. 1986;69:14.

[94] Trembly B SM. Double-blind clinical study of cannabidiol as a secondary anticonvulsant. Marijuana '90 International Conference on Cannabis and Cannabinoids. International Conference on Cannabis and Cannabinoids; 
1990 July 8-11; Kolympari, Crete.: International Association for Cannabinoid Medicines; 1990.

[95] Devinsky O, Marsh E, Friedman D, et al. Cannabidiol in patients with treatmentresistant epilepsy: an open-label interventional trial. Lancet neurology. 2016;15:270-8.

[96] Devinsky O, Sullivan J, Friedman D, et al. Efficacy and safety of Epidiolex (cannabidiol) in children and young adults with treatment-resistant epilepsy: Initial data from an expanded access progra. American Epilepsy Society annual meeting. Seattle, WA2014. 